\title{
Interference-free Determination of Indole-3-Acetic Acid in Two Real Systems Using Second-order Calibration Method Coupled with Excitation-emission Matrix Fluorescence
}

\author{
Xiu-Fang YAN, Hai-Long Wu, ${ }^{\dagger}$ Xiang-Dong QING, Yan-Mei Sun, and Ru-Qin Yu \\ State Key Laboratory of Chemo/Biosensing and Chemometrics, College of Chemistry and Chemical \\ Engineering, Hunan University, Changsha 410082, P. R. China
}

\begin{abstract}
In this work, a simple and practicable method that combines excitation-emission matrix (EEMs) fluorescence with a second-order calibration method based on parallel factor analysis-alternative least-squares (PARAFAC-ALS) algorithm was developed for the direct interference-free determination of indole-3-acetic acid (IAA) in two real systems, coconut water $(\mathrm{CW})$ and coconut milk $(\mathrm{CM})$. Although the excitation and emission profiles of IAA heavily overlapped with that of unknown interferents in the complex real systems, the actual contents and satisfactory recoveries were still obtained successfully. The contents of IAA in CW and CM were $10.8 \pm 0.3$ and $4.9 \pm 0.2 \mu \mathrm{g} \mathrm{mL}^{-1}$, respectively, which were consistent with those reported by LC-MS/MS assays in the reference material. The average spike recoveries of IAA in the validation set based on $\mathrm{CW}$ and $\mathrm{CM}$ were $102.1 \pm 3.2$ and $98.0 \pm 1.9 \%$, respectively. In addition, routine experiments were performed for establishing the validity of the assay to internationally accepted criteria.
\end{abstract}

Keywords IAA, EEMs, second-order calibration, PARAFAC-ALS

(Received November 19, 2013; Accepted February 12, 2014; Published April 10, 2014)

\section{Introduction}

Indole-3-acetic acid (IAA, Fig. 1) is the most common, naturally occurring plant hormone of the auxin class, and it possesses high activity even at low concentrations in plants. ${ }^{1,2}$ IAA has many different effects, as all auxins do, such as inducing cell elongation and cell division which are essential for plant growth and development. On a larger scale, IAA serves as the signaling molecule necessary for development of plant organs and coordination of growth. ${ }^{2-4}$ IAA exists in many kinds of vegetables and fruits, but the intake of IAA from cooked vegetables is very low because IAA easily decomposes open exposure to UV-light, heat and oxygen. However, without cooking or drying, IAA exists in plants at a concentration of its natural content. According to Motojima's research, IAA as a natural metabolite of the amino acid tryptophan in mammals with the aid of monoamine oxidase, ${ }^{5}$ has no obvious effect on the healthy organism, but can cause discomfort in mammals suffering from liver and kidney disease. ${ }^{6}$ The intake of IAA may cause proximal tubular injury via hOAT1-mediated (human renal organic anion transporter 1) uptake in patients with kidney disease. Therefore, it is essential to quantitatively determine IAA in fruits and foodstuff to minimize injury to patients with progressive renal disease and to assure correct utilization of natural resources.

Several analytical methods have been developed for the determination of IAA in these matrices, such as differential

$\dagger$ To whom correspondence should be addressed.

E-mail: hlwu@hnu.edu.cn pulse voltammetry (DPV), ${ }^{7}$ high performance liquid chromatography (HPLC), ${ }^{8}$ liquid chromatography-electrospray tandem mass spectrometry (LC-ESI-MS/MS), ${ }^{9,10}$ and hollow fiber-based liquid-liquid-liquid microextraction combined with high performance liquid chromatography-ultraviolet detection (HF-LLLME/HPLC-UV). ${ }^{11}$ However, these methods are usually accompanied by time-consuming and material-consuming procedures and also are a cause of environmental pollution. Hence, a simple and practicable method is expected for the efficient and accurate determination of IAA in plants. In this work, IAA in coconut water $(\mathrm{CW})$ and its product coconut milk (CM) were successfully determined by using excitationemission fluorescence in combination with the second-order calibration method. Furthermore, in order to test the reproducibility of the proposed method, inter- and intra-day experiments were repeated within three days.

\section{Theory}

Trilinear component model

The trilinear decomposition algorithm is suitable for three-<smiles>O=C(O)c1c[nH]c2ccccc12</smiles>

Fig. 1 Chemical structure formula of indole-3-acetic acid (IAA). 
way data sets $(\underline{\mathbf{X}})$ that satisfy the trilinear structure, which could be constructed from several two-way data or directly produced by luminoscope or hyphenated instruments (such as EEMs, HPLC-DAD). The noted trilinear component model proposed by Harshman, Caroll and Chang is one of the most acceptable models in the field of chemometrics. ${ }^{12,13}$ The trilinear component model could be described as follows:

$$
x_{\mathrm{ijk}}=\sum_{\mathrm{n}=1}^{\mathrm{N}} a_{\mathrm{in}} b_{\mathrm{jn}} c_{\mathrm{kn}}+e_{\mathrm{ijk}} \quad(i=1, \ldots, I ; j=1, \ldots, k=1, \ldots, K)
$$

where $x_{\mathrm{ijk}}$ is the $(i, j, k)$ th element of $\underline{\mathbf{X}}$, and $i, j, k$ represent three different modes of the three-way data array, respectively. In this work, $x_{\mathrm{ijk}}$ is the fluorescence intensity of sample $k$ at excitation wavelength $i$ and emission wavelength $j, N$ is the total number of factors that can be detected in the system, which should be considered as the total number of detectable species, containing the component(s) of interest and the background as well as the uncalibrated interferent(s). $a_{\text {in }}$ is the element $(i, n)$ of $I \times N$ matrix $\mathbf{A}, b_{\mathrm{jn}}$ is element $(j, n)$ of $J \times N$ matrix $\mathbf{B}$, and $c_{\mathrm{kn}}$ is the element of $K \times N$ matrix $\mathbf{C}$, and $e_{\mathrm{ijk}}$ is the residual element of an $I \times J \times K$ three-way residual array $\underline{\mathbf{E}}$. In matrix form, the trilinear component model can be expressed as:

$$
\begin{array}{ll}
\mathbf{X}_{\mathrm{I} \times \mathrm{JK}}=\mathbf{A}(\mathbf{C} \odot \mathbf{B})^{\mathrm{T}}+\mathbf{E}_{\mathrm{I} \times \mathrm{JK}}, & i=1,2, \ldots, I \\
\mathbf{X}_{\mathrm{I} \times \mathrm{KI}}=\mathbf{B}(\mathbf{A} \odot \mathbf{C})^{\mathrm{T}}+\mathbf{E}_{\mathrm{J} \times \mathrm{KI}}, & j=1,2, \ldots, J \\
\mathbf{X}_{\mathrm{K} \times \mathrm{IJ}}=\mathbf{C}(\mathbf{B} \odot \mathbf{A})^{\mathrm{T}}+\mathbf{E}_{\mathrm{K} \times \mathrm{IJ}}, & k=1,2, \ldots, K
\end{array}
$$

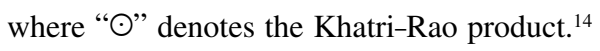

\section{PARAFAC-ALS algorithm}

The parallel factor analysis-alternative least-squares (PARAFAC-ALS) algorithm is commonly carried out through an alternative least-squares (ALS) minimization scheme, which was originated from psychometrics and first proposed by Harshman under that name in $1970 .{ }^{12}$ At the same time, Carol and Chang studied it under the name of canonical decomposition (CANDECOMP). ${ }^{13}$ According to Eqs. (2a) - (2c), the general updates of the PARAFAC-ALS procedure can be repeated as follows:

$$
\begin{aligned}
& \mathbf{A}=\mathbf{X}_{\mathrm{I} \times \mathrm{JK}}\left[(\mathbf{C} \odot \mathbf{B})^{\mathrm{T}}\right]^{+}, \\
& \mathbf{B}=\mathbf{X}_{\mathrm{J} \times \mathrm{KI}}\left[(\mathbf{A} \odot \mathbf{C})^{\mathrm{T}}\right]^{+}, \\
& \mathbf{C}=\mathbf{X}_{\mathrm{K} \times \mathrm{II}}\left[(\mathbf{B} \odot \mathbf{A})^{\mathrm{T}}\right]^{+},
\end{aligned}
$$

where '+' denotes the Moore-Penrose inverse. $\mathbf{C}$ is calculated by initializing $\mathbf{A}$ and $\mathbf{B}$ randomly by using Eq. (3c), then $\mathbf{A}$ and B are computed by Eqs. (3a) and (3b), respectively. ${ }^{15}$

\section{Figures of merit}

Figures of merit is of significant importance in developing and assessing the reliability of analytical methodologies, such as net analyte signal (NAS), ${ }^{16,17}$ selectivity (SEL), sensitivity (SEN), ${ }^{17}$ the limit of detection (LOD), the limit of quantification (LOQ) ${ }^{18}$ and the root-mean-square error of prediction (RMSEP). ${ }^{19}$ The SEN and SEL for a particular analyte are presented as follows: ${ }^{16,17}$

$$
\begin{aligned}
& \mathrm{SEN}=k\left\{\left[\left(\mathbf{B}^{\mathrm{T}} \mathbf{B}\right)^{-1}\right]_{\mathrm{nn}}\left[\left(\mathbf{C}^{\mathrm{T}} \mathbf{C}\right)^{-1}\right]_{\mathrm{nn}}\right\}^{-1 / 2}, \\
& \mathrm{SEL}=\left\{\left[\left(\mathbf{B}^{\mathrm{T}} \mathbf{B}\right)^{-1}\right]_{\mathrm{nn}}\left[\left(\mathbf{C}^{\mathrm{T}} \mathbf{C}\right)^{-1}\right]_{\mathrm{nn}}\right\}^{-1 / 2},
\end{aligned}
$$

where $n n$ designates the $(n, n)$ element of a matrix, $k$ is the total signal for component $n$ at unit concentration and it represents a parameter for converting scores to concentrations. The LOD and LOQ are calculated as the Eqs. (6) and (7) according to the literature: ${ }^{18}$

$$
\begin{aligned}
& \mathrm{LOD}=3.3 s(0) \\
& \mathrm{LOQ}=10 s(0)
\end{aligned}
$$

where $s(0)$ is the standard deviation in the concentration estimated from three different background blank samples.

The root-mean-square error of prediction can be described as:

$$
\mathrm{RMSEP}=\left[\{1 /(M-1)\} \sum_{\mathrm{m}=1}^{\mathrm{M}}\left(c_{\mathrm{act}, \mathrm{m}}-c_{\text {pred, } \mathrm{m}}\right)^{2}\right]^{1 / 2}
$$

where $M$ is the number of prediction samples, and $c_{\text {act, } m}$ and $c_{\text {pred, } m}$ are correspond to the actual added and predicted concentration of analytes of interest, respectively.

\section{Experimental}

\section{Reagents and chemicals}

IAA (>99\%) was purchased from Aladdin Regent (Shanghai, China), stored and used as soon as received. All other chemicals used were of analytical grade and all of the solutions were prepared with ultra-pure grade water. Ultra-pure grade water with parameter of $18.20 \mathrm{M} \Omega \mathrm{cm}^{-1}$ was prepared using a Milli-Q water purification system (Millipore, USA). Coconut water and coconut milk were purchased from local markets. A buffer solution of $\mathrm{pH} 5.6$ was prepared by using sodium hydrogen phosphate and citric acid. IAA was weighed accurately $10.36 \mathrm{~g}$, dissolved in $10.0 \mathrm{~mL}$ alcohol, then transferred to a $50.0-\mathrm{mL}$ brown glass volumetric flask and diluted with ultrapure water to the mark to obtain a stock solution with a concentration of $207.2 \mu \mathrm{g} \mathrm{mL}^{-1}$. It was then wrapped in silver paper and stored in darkness at $4^{\circ} \mathrm{C}$. A working solution of coconut water was prepared by diluting $5.0 \mathrm{~mL}$ fresh coconut water with ultrapure water to $250.0 \mathrm{~mL}$, and was not subjected to any other pretreatment except filtering through a $0.45-\mu \mathrm{m}$ cellulose membrane filter. The working solution of coconut milk was obtained by handling $5.0 \mathrm{~mL}$ coconut milk in the same manner as the coconut water samples.

\section{Apparatus}

All measurements of the fluorescence signal were performed on a fluorescence spectrophotometer (F-7000, Hitachi, Japan) equipped with a continuous Xenon arc lamp and a computer. EEMs were record every $2.0 \mathrm{~nm}$ over the range of $202.0-330.0$ and $250.0-450.0 \mathrm{~nm}$ for excitation and emission wavelengths, respectively. The excitation and emission slit widths were both $5.0 \mathrm{~nm}$ and the working voltage was $600 \mathrm{~V}$. All computer programs were written in-house in the MATLAB environment, and all programs were run on an IBM compatible microcomputer.

\section{Experimental process}

A calibration set of seven samples (C01 - C07) was constructed with concentrations designed as an arithmetic progression in the range of $8.0-80.0 \mathrm{ng} \mathrm{mL}^{-1}$, within the linear range of IAA of $6.0-500.0 \mathrm{ng} \mathrm{mL}^{-1}$ and with a correlation coefficient of $R^{2}=$ 0.997 . Then one group of three actual samples of coconut water (CW01-CW03, $1.0 \mathrm{~mL} \mathrm{CW}$ working solution diluted to $10.0 \mathrm{~mL}$ ) and another coconut milk (CM01-CM03, $0.9 \mathrm{~mL}$ 
CM working solution diluted to $10.0 \mathrm{~mL}$ ) group were prepared, respectively. What is more, five validation samples based on these two actual systems (VA01 - VA05 for coconut water and VB01 - VB05 for coconut milk) were constructed, respectively. The validation samples were obtained by spiking IAA into the same amount of actual samples within the concentration of the calibration range by arithmetic progression. The concentrations of the calibration set are displayed in Table 1 and those for the other samples are shown in the columns 1,2, 5, and 6 of Table 2 .

\section{Results and Discussion}

Spectra characteristics of IAA and two corresponding samples Figure 2 presents the spectra contour map of IAA (sample C04), CW (CW01) and CM (CM01) which had been substituted Rayleigh and Raman scattering as fitted value (described in the next section). As can be seen from Fig. 2, the location of the

Table 1 Concentrations of seven calibration samples

\begin{tabular}{cc}
\hline Sample & IAA/ng mL $\mathrm{mL}^{-1}$ \\
\hline C01 & 8.0 \\
C02 & 20.0 \\
C03 & 32.0 \\
C04 & 44.0 \\
C05 06 & 56.0 \\
C06 & 68.0 \\
C07 & 80.0 \\
\hline
\end{tabular}

peak only presents a slight difference, the maximum emission peak of IAA, CW and CM are located at 360.0, 350.0 and $334.0 \mathrm{~nm}$, respectively. This shows that one or more interferents exist in these two actual systems and present seriously overlapped profiles with the spectra profile of IAA, which can be further verified in the next section. However, under this circumstance, without combining with the chemometric method, the traditional fluorescence method is not sufficient for directly quantifying the analytes of interest from these seriously overlapped and highly colinearity spectra. This could only be achieved with the aid of complex sample separation and extraction, which are both labor and resource intensive.

\section{Three-dimensional fluorescence spectra of IAA and the data processing}

The first-order Rayleigh scattering that partly overlaps with the signals of IAA and the Raman scattering that even runs throughout it makes the data analysis rather difficult. When selecting a rectangular area without Rayleigh and Raman scattering, it will result in losing a great deal of information of the samples or even lead to erroneous results. So a method for dealing with this problem was taken. ${ }^{20}$ Figure 3 demonstrates one part of the data treatment procedure, and Fig. 3(a) presents the original three-dimensional spectra of the calibration sample C04. The spectra then can be seen as Fig. 3(b) after substituting the first-order and second-order Rayleigh scattering and Raman scattering as missing, and Fig. 3(c) corresponds to the threedimensional spectra of $\mathrm{C} 04$ which had been handled by the interpolation method. ${ }^{20,21}$ All of the data obtained had been handled by the same data process by substituted Rayleigh and Raman scattering as fitted value using the interpolation method.

Table 2 Actual and validation samples and the results obtained from the PARAFAC-ALS algorithm

\begin{tabular}{rrrrrrrr}
\hline Sample & $\begin{array}{c}\text { Spiked/ } \\
\mathrm{ng} \mathrm{mL} \mathrm{mL}^{-1}\end{array}$ & $\begin{array}{c}\text { Found/ } \\
\mathrm{ng} \mathrm{mL}\end{array}$ & $\begin{array}{c}\text { Recovery } \\
\%\end{array}$ & Sample & $\begin{array}{c}\text { Spiked/ } \\
\mathrm{ng} \mathrm{mL}^{-1}\end{array}$ & $\begin{array}{c}\text { Found/ } \\
\mathrm{ng} \mathrm{mL}^{-1}\end{array}$ & $\begin{array}{c}\text { Recovery } \\
\%\end{array}$ \\
\hline VA01 & 8.0 & 29.6 & 99.6 & VB01 & 8.0 & 16.4 & 95.0 \\
VA02 & 26.0 & 48.6 & 103.7 & VB02 & 26.0 & 34.3 & 98.1 \\
VA03 & 44.0 & 66.2 & 101.3 & VB03 & 44.0 & 51.6 & 97.2 \\
VA04 & 62.0 & 87.8 & 106.7 & VB04 & 62.0 & 70.8 & 100.0 \\
VA05 & 80.0 & 101.0 & 99.2 & VB05 & 80.0 & 88.1 & 99.1 \\
CW01 & 0.0 & 22.0 & & CM01 & 0.0 & 8.3 & \\
CW02 & 0.0 & 21.9 & $21.6 \pm 0.6$ & CM02 & 0.0 & 9.2 & $8.8 \pm 0.5$ \\
CW03 & 0.0 & 21.0 & & CM03 & 0.0 & 8.8 & \\
\hline
\end{tabular}

a. Recovery $=($ found concentration - basic concentration $(\mathrm{CW})) /$ spiked concentration $\times 100 \%$. b. Recovery $=($ found concentration - basic concentration $(\mathrm{CM})) /$ spiked concentration $\times 100 \%$.

a

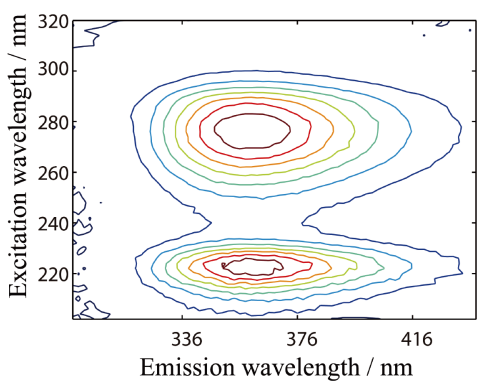

b

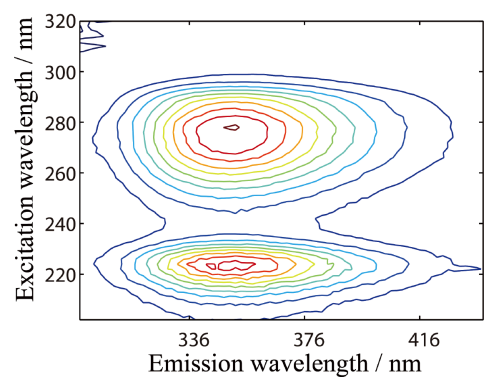

C

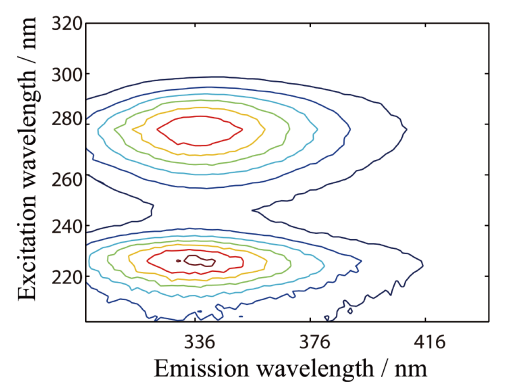

Fig. 2 The excitation and emission spectra of indole-3-acetic acid (IAA), coconut water (CW) and coconut milk (CM). (a) illustrates the contour map of excitation-emission spectra of pure IAA, (b) and (c) correspond to the excitation-emission fluorescence spectra of $\mathrm{CW}$ and $\mathrm{CM}$, respectively. 
In second-order calibration, estimation of the number of components is of great importance. In this work, the number of components $N=4$ was obtained from the core consistency diagnostic. 22 While considering that 1) the presence of noise and interferents in the target systems may affect the rank estimation process, and 2) two different and complex actual
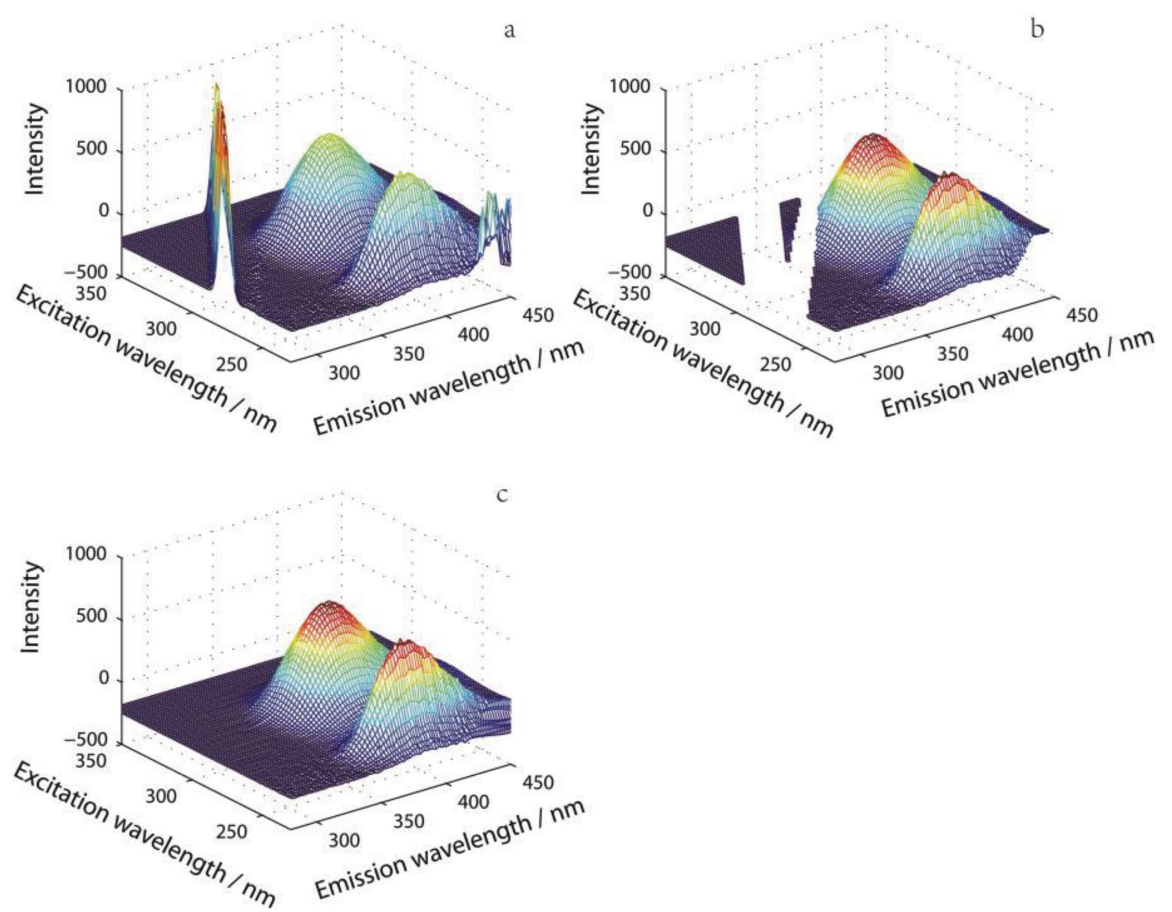

Fig. 3 Excitation-emission spectra of sample C04. (a) represents the original spectrum, (b) illustrates the spectrum after removal of the Rayleigh and Raman scattering, and (c) shows the spectrum had been handled by the interpolation method.
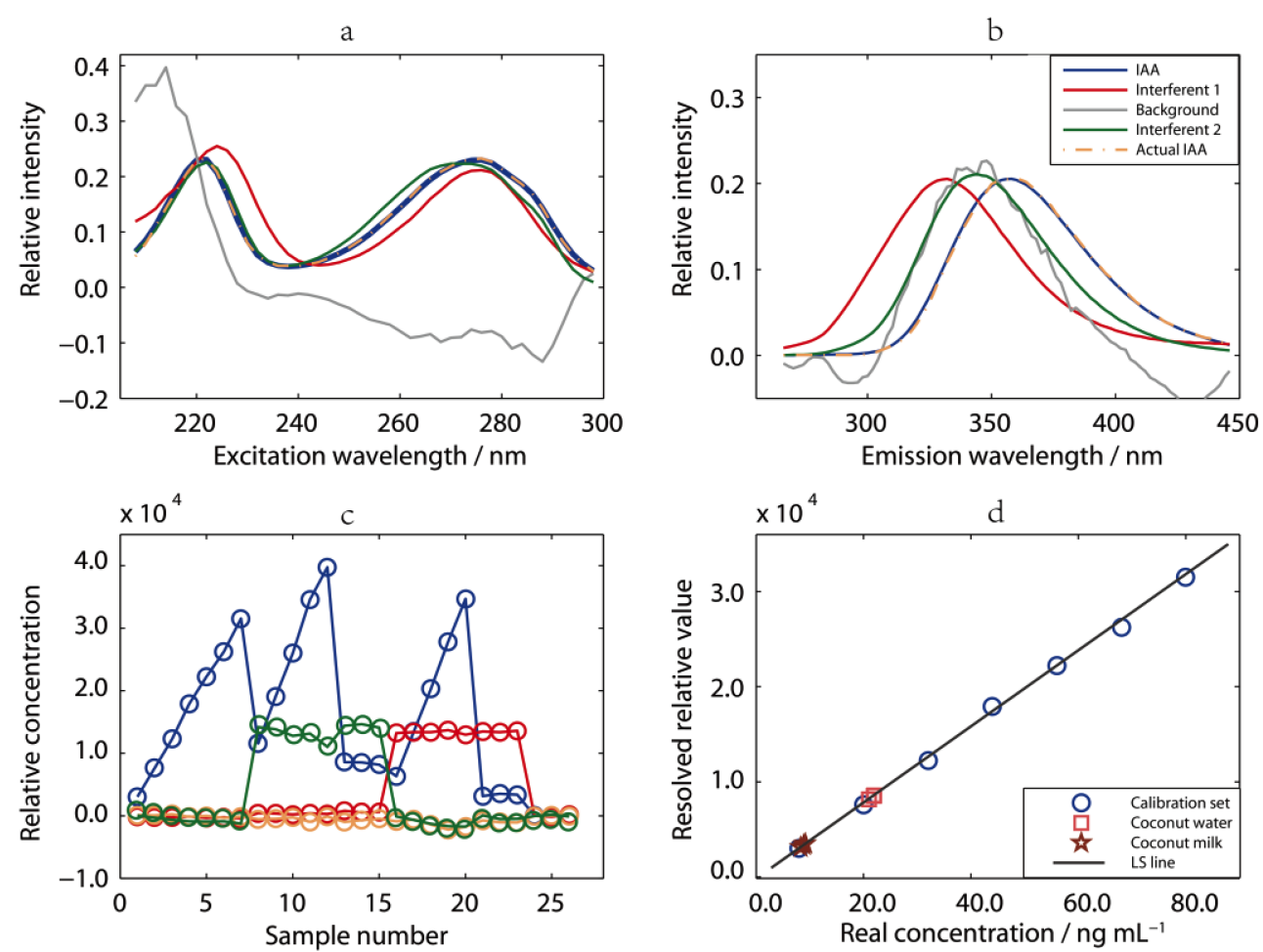

Fig. 4 Normalized resolved excitation and emission spectra and relative concentration profile obtained from the PARAFAC-ALS algorithm with $N=4$. (a) Excitation spectra, (b) emission spectra, (c) relative concentration profile, (d) the relationship between the actual concentration and the resolved relative value. 
systems were analyzed together. Finally, the number of components $N=4$ was selected for the quantitative analysis of IAA in the two actual systems by using the PARAFAC-ALS algorithm. The normalized excitation and emission profiles, as well as the relative concentration information are clearly illustrated in Fig. 4. Although the excitation and emission spectra of these interferents seriously overlapped with the spectra of IAA, the quantitative analysis of IAA in these two actual systems was still achieved successfully due to the 'second-order advantages' of the second-order calibration method. ${ }^{23-27}$

Figures 4(a) and 4(b) show the resolved IAA excitation and emission spectral profiles and the corresponding actual spectra profiles of IAA acquired by normalization practical measured spectra. The resolved spectra of IAA (the blue line) dovetail extremely well with the actual spectra profile of IAA (the bright orange dotted line), both for the excitation and emission spectra. Figure 4(c) presents the relative concentration profile of IAA, background and interferents. The results should be acceptable for the uniqueness of the trilinear decomposition, the high consistency between the resolved and actual spectra fully guaranteed the uniqueness of the third mode, namely, the resolved concentration profile of IAA. Due to the "second-order advantages' of second-order calibration based on the PARAFACALS algorithm, the spectra profile of IAA can be resolved from the mixed spectra data, which contained unknown interferents and background. Figure 4(d) illustrates the relationship between the resolved concentration profile and the actual concentration. The contents of IAA in CW and CM located perfectly on the regression curve (LS line) within the range of calibration concentration, which suggests the content of the IAA in CW and $\mathrm{CM}$ has been correctly detected.

\section{The actual contents of IAA in real samples}

Regression with the actual concentration and the resolved concentration profile of IAA in the calibration set achieved a calibration curve with correlative coefficient of $R^{2}=0.999$, which indicated that the IAA possesses good linear correlation in the range of $8.0-80.0 \mathrm{ng} \mathrm{mL}^{-1}$. Table 2 summarizes the spiked concentration and the found concentration of IAA in CW and CM samples. The average contents of IAA in CW and CM were $21.6 \pm 0.6$ and $8.8 \pm 0.5 \mathrm{ng} \mathrm{mL}^{-1}$, respectively, and when converted back to the actual contents of IAA in CW and CM, according to the dilution ratio, turned into $10.8 \pm 0.3$ and $4.9 \pm 0.2 \mu \mathrm{g} \mathrm{mL}^{-1}$, respectively. In addition, the average recovery of the validation set based on CW was of $102.1 \pm$ $3.2 \%$, and for $\mathrm{CM}$ was $98.0 \pm 1.9 \%$, which suggested that the results were quite reliable.

\section{Comparison of analytical results}

The comparison of the results of the actual contents of IAA in CW obtained from different works following different analytical approaches was taken. In this work, the contents of IAA in CW of $10.8 \pm 0.3 \mu \mathrm{g} \mathrm{mL}^{-1}$ was quite similar to $0.122 \mu \mathrm{M}$ $\left(21.38 \mu \mathrm{g} \mathrm{mL}^{-1}\right)$ obtained by the LC-MS/MS assay by Ma et al. ${ }^{10}$ the tiny difference falls within the acceptable range attributable to using different coconut materials. Due to the power of the 'second-order advantages' of the second-order calibration method used in this work, the experiment has been simplified to a great extent. In this work, except for filtering through a $0.45-\mu \mathrm{m}$ cellulose membrane filter for removing solids presented in $\mathrm{CW}$ and $\mathrm{CM}$, no other pretreatments were adopted. However, for the LC-MS/MS method, the complex pretreatment process and the loss of analytes of interest during the chemical extraction and purification are inevitable.
Table 3 Figures of merit of determination of IAA using the PARAFAC-ALS algorithm

\begin{tabular}{ccccccc}
\hline SEL & $\begin{array}{c}\mathrm{SEN} / \\
\mathrm{mL} \mathrm{ng}^{-1}\end{array}$ & $\begin{array}{c}\mathrm{LOD} / \\
\mathrm{ng} \mathrm{mL}^{-1}\end{array}$ & $\begin{array}{c}\mathrm{LOQ} / \\
\mathrm{ng} \mathrm{mL}^{-1}\end{array}$ & $\begin{array}{c}\mathrm{RMSEP}^{2} \\
\mathrm{ng} \mathrm{mL}^{-1}\end{array}$ & Method & Ref. \\
\hline 0.07 & 27.35 & 0.79 & 2.39 & 1.53 & EEMs & This work \\
$\mathrm{NG}^{\mathrm{a}}$ & $\mathrm{NG}$ & 220.8 & 669.2 & $\mathrm{NG}^{\mathrm{a}}$ & LC-MS/MS & 10 \\
\hline
\end{tabular}

a. No given.

Table 4 Results of inter- and intra- day experiments obtained from PARAFAC-ALS

\begin{tabular}{ccccccc}
\hline & & \multicolumn{2}{c}{ Inter-day } & & \multicolumn{2}{c}{ Intra-day } \\
\cline { 6 - 7 } \cline { 5 - 6 } Sample & $\begin{array}{c}\text { Added/ } \\
\text { ng mL }\end{array}$ & $\begin{array}{c}\text { Recovery } \\
\text { average } \pm \text { SD }^{\mathrm{a}}, \%\end{array}$ & $\begin{array}{c}\mathrm{RSD}^{\mathrm{b}}, \\
\%\end{array}$ & & $\begin{array}{c}\text { Recovery } \\
\text { average } \pm \mathrm{SD}^{\mathrm{a}}, \%\end{array}$ & $\begin{array}{c}\mathrm{RSD}^{\mathrm{b}}, \\
\%\end{array}$ \\
\hline \multirow{2}{*}{$\mathrm{CW}$} & 8.0 & $105.7 \pm 8.7$ & 8.3 & & $104.2 \pm 7.5$ & 7.2 \\
& 26.0 & $103.4 \pm 7.3$ & 7.1 & & $99.1 \pm 5.4$ & 5.4 \\
& 44.0 & $100.2 \pm 1.8$ & 1.8 & & $103.5 \pm 5.8$ & 5.6 \\
& 62.0 & $99.4 \pm 4.7$ & 4.7 & & $101.7 \pm 5.8$ & 5.7 \\
& 80.0 & $99.5 \pm 5.8$ & 5.8 & & $100.5 \pm 3.3$ & 3.3 \\
$\mathrm{CM}$ & 8.0 & $96.7 \pm 3.2$ & 3.3 & & $99.3 \pm 1.4$ & 1.4 \\
& 26.0 & $97.4 \pm 1.0$ & 1.0 & & $100.2 \pm 1.8$ & 1.9 \\
& 44.0 & $96.5 \pm 1.2$ & 1.2 & & $99.3 \pm 3.8$ & 3.8 \\
& 62.0 & $95.5 \pm 2.8$ & 3.0 & & $97.5 \pm 4.2$ & 4.3 \\
& 80.0 & $91.6 \pm 0.1$ & 3.3 & $93.8 \pm 2.2$ & 2.4 \\
\hline
\end{tabular}

a. $\mathrm{SD}=\sqrt{\sum_{\mathrm{i}=1}^{\mathrm{n}}\left(x_{\mathrm{i}}-\bar{x}\right)^{2} / n-1}, \mathrm{~b} . \mathrm{RSD}=\frac{\mathrm{SD}}{\bar{x}} \times 100$.

Furthermore, from the data demonstrated in Table 3, we can come to the conclusion that EEM is superior to LC-MS/MS for the determination of IAA in LOD and LOQ.

\section{Figures of merit}

In order to evaluate the performance of the proposed method, figures of merit including SEL, SEN, LOD, LOQ and RMSEP, based on the second-order calibration algorithm PARAFAC have been studied, and the results are summarized in Table 3 . Compared with the LC-MS/MS method mentioned above (in which the LOD value was $\left.3.8 \mu \mathrm{M}\left(220.8 \mathrm{ng} \mathrm{mL}^{-1}\right)\right)$, the LOD was significantly lower in this work. Moreover, other satisfactory figures of merit were also acquired here, which indicated that the proposed method would be effective for quantifying analytes of interest even in the presence of other unknown and uncalibrated fluorescent components.

\section{Inter- and intra-assay accuracy and precision}

In order to test inter- and intra-assay accuracy and precision of the proposed method, inter- and intra-day experiments were carried out by assaying five replicates of each of the five concentrations of validation samples on two real systems (CW and $\mathrm{CM}$ ) within three days. The results of average recovery \pm standard deviation (SD) and relative standard deviation (RSD, $\%)$ are summarized in Table 4 . The average RSDs of the validation set based on $\mathrm{CM}$ and $\mathrm{CW}$ were about 5.5 and $2.6 \%$, respectively. All other values obtained were also well within internationally recognized acceptance criteria for assay validations, which indicated the proposed method possesses good reproducibility. 


\section{Conclusions}

In this paper, the combination of excitation-emission fluorescence matrix with second-order calibration method based on the PARAFAC-ALS algorithm was developed and validated for the successful determination of IAA in two different real systems simultaneously. The proposed method was fully validated by studies on the average recoveries of validation sets based on real system samples, the reproducibility of the proposed method and figures of merit. The method used in this paper is quite simple, rapid, convenient, reliable and practical, and possesses high selectivity and capability for the determination of analytes of interest in different real samples. The proposed method provides an excellent alternative for the direct determination of IAA in complex actual systems, without the need for analytical separation.

\section{Acknowledgements}

The authors gratefully acknowledge financial support from the National Natural Science Foundation of China (Grant No. 21175041) and the National Basic Research Program (No. 2012CB910602) as well as the Foundation for Innovative Research Groups of NSFC (Grant No. 21221003).

\section{References}

1. M. P. De Mello, S. M. De Toledo, M. Haun, G. Cilento, and N. Duran, Biochemistry, 1980, 19, 5270.

2. P. J. Davies, "The Plant Hormones: Their Nature, Occurrence, and Functions", 2010, Plant Hormones, Springer Netherland, New York, 1.

3. H. Tanaka, P. Dhonukshe, P. Brewer, and J. Friml, Cell. Mol. Life Sci., 2006, 63, 2738.

4. S. Vanneste and J. Friml, Cell, 2009, 136, 1005.

5. M. J. Sarrias, P. Cabré, E. Martínez, and F. Artigas, J. Neurochem., 1990, 54, 783.

6. M. Motojima, A. Hosokawa, H. Yamato, T. Muraki, and T.
Yoshioka, Br. J. Pharmacol., 2002, 135, 555.

7. J. Bulíčková, R. Sokolová, S. Giannarelli, and B. Muscatello, Electroanalysis, 2013, 25, 303.

8. K. Hoenicke, T. J. Simat, H. Steinhart, H. J. Köhler, and A. Schwab, J. Agric. Food Chem., 2001, 49, 5494.

9. A. Durgbanshi, V. Arbona, O. Pozo, O. Miersch, J. V. Sancho, and A. Gomez-Cadenas, J. Agric. Food Chem., 2005, 53, 8437.

10. Z. Ma, L. Ge, A. S. Lee, J. W. H. Yong, S. N. Tan, and E. S. Ong, Anal. Chim. Acta, 2008, 610, 274.

11. Y. Wu and B. Hu, J. Chromatogr. A, 2009, 1216, 7657.

12. R. A. Harshman, UCLA Working Papers in Phonetics, 1970, 16,1 .

13. J. D. Carroll and J.-J. Chang, Psychometrika, 1970, 35, 283.

14. C. G. Khatri and C. R. Rao, Sankhya, Ser. A, 1968, 30, 167.

15. A.-L. Xia, H.-L. Wu, S.-H. Zhu, Q.-J. Han, Y. Zhang, and R.-Q. Yu, Anal. Sci., 2008, 24, 1171.

16. A. Lorber, Anal. Chem., 1986, 58, 1167.

17. C. Ho, G. Christian, and E. Davidson, Anal. Chem., 1980, $52,1071$.

18. R. Boqué, J. Ferré, N. K. M. Faber, and F. X. Rius, Anal. Chim. Acta, 2002, 451, 313.

19. A. Lorber, K. Faber, and B. R. Kowalski, Anal. Chem., 1997, 69, 1620.

20. M. Bahram, R. Bro, C. Stedmon, and A. Afkhami, $J$. Chemom., 2006, 20, 99.

21. Y. Li, H.-L. Wu, Y.-J. Yu, S. Zhang, Y. Chen, D.-Z. Tu, C.-C. Nie, H. Xu, and R.-Q. Yu, Anal. Methods (UK), 2012, 4, 3987.

22. R. Bro and H. A. L. Kiers, J. Chemom., 2003, 17, 274.

23. P. C. Damiani, A. J. Nepote, M. Bearzotti, and A. C. Olivieri, Anal. Chem., 2004, 76, 2789.

24. E. Fuentes, M. E. Báez, M. Bravo, C. Cid, and F. Labra, Food Anal. Methods, 2012, 5, 1311.

25. A.-L. Xia, H.-L. Wu, D.-M. Fang, Y.-J. Ding, L.-Q. Hu, and R.-Q. Yu, Anal. Sci., 2006, 22, 1189.

26. H. L. Wu, M. Shibukawa, and K. Oguma, J. Chemom., 1998, $12,1$.

27. D.-M. Fang, H.-L. Wu, Y.-J. Ding, L.-Q. Hu, A.-L. Xia, and R.-Q. Yu, Talanta, 2006, 70, 58. 